\title{
Efficacy and acceptability of parent-only group cognitive behavioral intervention for treatment of anxiety disorder in children and adolescents: a meta-analysis of randomized controlled trials
}

\author{
Bangmin Yin ${ }^{1,2,3}$, Teng Teng ${ }^{2,3}$, Lyu Tong ${ }^{4}$, Xuemei Li, ${ }^{2,3}$ Li Fan ${ }^{2,3}$, Xinyu Zhou ${ }^{5^{*}}$ and Peng Xie L $^{2,3^{*}}$ (1)
}

\begin{abstract}
Background: Anxiety disorder is the most prevalent mental disorder among children and adolescents, causing significant psychosocial problems and physical health conditions. Cognitive behavioral therapy (CBT) is an effective treatment for anxiety disorder in children and adolescents. And parent-only CBT is an alternative treatment for childhood anxiety disorder, which includes psychologists and parents rather than children in the treatment. As a new type of CBT, parent-only CBT has some advantages. However, it remains unclear whether parent-only CBT interventions are effective for treating children and adolescents with anxiety disorder.
\end{abstract}

Methods: In this study, we evaluated the efficacy (the mean change scores of the anxiety rating scale from baseline to post-treatment, standardized mean difference SMD) and acceptability (the proportion of patients in the treatment group who withdrew from treatment early for any reason, risk ratios RRs) of parent-only cognitive behavioral therapy (CBT) for children and adolescents with anxiety disorder. We searched electronic databases, including PubMed, Cochrane Library, Embase, Web of Science, ProQuest, and PsycINFO from inception to June 2019. We included randomized controlled trials (RCTs) comparing parent-only CBT either with waitlist (WL), or CBT with parents in children and adolescents with anxiety disorder.

Results: Finally, six RCTs with 407 participants were included in the meta-analyses. In terms of efficacy, pooled analyses indicated that parent-only CBT was significantly more effective than WL for reducing anxiety symptoms with SMD of $-0.72(95 \% \mathrm{Cl}-1.41$ to $-0.03, p=0.04)$, and more remission rate with RR of $4.33(37.96 \%$ vs. 6.85 , $95 \% \mathrm{Cl} 1.82$ to $10.27, p=0.0009)$ at post-treatment. And our analyses showed no evidence that parent-only CBT had significantly greater efficacy than CBT with parents with SMD of $0.21(95 \% \mathrm{Cl}-0.09$ to $0.50, p=0.17)$. Acceptability in the parent-only CBT group was not significantly different to the WL group with RR of 0.92 ( $95 \% \mathrm{Cl}$ (Continued on next page)

\footnotetext{
*Correspondence: xinyu973@126.com; xiepeng973@126.com

${ }^{5}$ Department of Psychiatry, The First Affiliated Hospital of Chongqing Medical University, No. 1 Youyi Road, Chongqing 400016, China

${ }^{2}$ Department of Neurology, The First Affiliated Hospital of Chongqing Medical University, Chongqing, China

Full list of author information is available at the end of the article
} 4.0 International License, which permits use, sharing, adaptation, distribution and reproduction in any medium or format, as long as you give appropriate credit to the original author(s) and the source, provide a link to the Creative Commons licence, and indicate if changes were made. The images or other third party material in this article are included in the article's Creative Commons licence, unless indicated otherwise in a credit line to the material. If material is not included in the article's Creative Commons licence and your intended use is not permitted by statutory regulation or exceeds the permitted use, you will need to obtain permission directly from the copyright holder. To view a copy of this licence, visit http://creativecommons.org/ licenses/by/4.0/. The Creative Commons Public Domain Dedication waiver (http://creativecommons.org/publicdomain/zero/1. 0/) applies to the data made available in this article, unless otherwise stated in a credit line to the data. 
(Continued from previous page)

0.52 to $1.62, p=0.77$ ), and was significantly worse than in the CBT with parents group with RR of 1.93 (95\% Cl 1.05 to $3.57, p=0.03)$.

Conclusions: Current evidence indicates that parent-only CBT can be an alternative and acceptable intervention for treating children and adolescents with anxiety disorder.

Keywords: Anxiety, Parent-only CBT, Meta-analysis, Child, Adolescent

\section{Background}

Anxiety disorder is the most prevalent mental disorder among children and adolescents. Internationally, $6.5 \%$ of all children and adolescents meet the diagnostic criteria for anxiety disorder at least once in their life [1]. Anxiety disorder causes significant psychosocial problems, including impaired academic and social competence, and can lead to physical health problems [2, 3].

Cognitive behavioral therapy (CBT) is an effective treatment for childhood anxiety disorder. And parentonly CBT is an alternative treatment for childhood anxiety disorder, which includes psychologists and parents rather than children in the treatment. By previous study, we reported meta-analysis about child-CBT for anxiety disoder and noticed parent-only CBT for children anxiety is an interesting treatment which have unique advantages, especially for young children. So on the basis, we did this research focused on parent-only CBT [4-10]. As one type of CBT used with children and adolescents, CBT with parents has been demonstrated to be effective for treating children and adolescents with anxiety $[5,11,12]$. However, child-focused CBT has several disadvantages. First, language and cognitive competence are major obstacles, especially for young children [13]. Stigma associated with receiving mental health intervention is another significant obstacle for children $[14,15]$, as children are more likely to be stigmatized by others for help-seeking behaviors, compared with parents [14]. Furthermore the importance of the family environment and parenting style factors have been identified in previous studies focused on the etiology of childhood anxiety disorders [15].

To respond to these challenges, research into parent-focused interventions for childhood anxiety disorder is increasing. This type of intervention has the potential to avoid the problems mentioned above. However, it remains unclear whether parentonly CBT interventions are effective for treating children and adolescents with anxiety disorder, and previous studies have produced conflicting findings $[13,16-18]$. Therefore, we designed a conventional meta-analysis to determine the effectiveness of parent-only CBT to treat anxiety disorder in children and adolescents, compared with WL or CBT with parents as comparison group.

\section{Methods}

\section{Data sources and searches}

PRISMA guidelines [19] were used for conducting this meta-analysis. We conducted a systematic search of six electronic databases: PubMed, Cochrane Library, Embase, Web of Science, ProQuest, and PsycINFO from inception to June 2019. No restrictions were used regarding language. The keywords included: anxiety OR anxious OR phobic OR fear OR fears OR phobia OR phobias, and adolesc* OR child* OR boy* OR girl* OR juvenil* OR minors OR paediatri* OR pediatri* OR pubescen* OR school* OR student* OR teen* OR young, and behavio* OR cogniti* OR CBT OR famil* OR "contingency management", and parent* OR mother OR father, and random* OR allocate* OR assign* OR "cross over*" OR crossover* OR controlled. More details of literature search reports can be found in Additional file 1. Furthermore, to identify additional eligible randomized controlled trials (RCTs), the reference lists of relevant studies were scanned.

\section{Studies selection and comparisons design}

To determine appropriate studies for our meta-analysis exploring the efficacy and acceptability for treatment of children and adolescents with anxiety disorder, we selected studies according to the following criteria: (1) we included any RCTs investigating the application of parent-only CBT for the treatment of anxiety disorder in children and adolescents, and comparing parenr-only CBT with WL or CBT with parents; (2) we included studies with children and adolescents who were under the age of 18; (3) All participants met a primary diagnosis of a current anxiety disorder, conforming to standardized diagnostic criteria (DSM-IV and K-SADS-PL) [20-22]. to enhance the reliability of our conclusions, we designed two comparisons that complemented each other. One group compared parent-only CBT with WL as a control group, while another group compared parent-only CBT with CBT with parents. In order to focus on anxiety disorder, we excluded studies in which more than $20 \%$ of participants had a primary diagnosis of other mental disorders. We adapt relatively strict criterion about including children with anxiety disorder, so studies in which patients were only described with anxiety symptoms rather than confirmed diagnoses were 
excluded. Because anxiety disorder is frequently comorbid other mental disorders, we did not exclude studies in which participants had a secondary diagnosis of comorbid psychiatric diseases such as major depression and attention deficit hyperactivity disorder. For avoiding influence of drug, trials were excluded if more than 20\% of patients took psychotropic drugs. Previous literature introduced Primary Care Behavioral Health (PCBH) model designed by the Veteran's Administration and the Bureau of Primary Care [23]. The PCBH model recomends that the patients who came to the first consult with the psychologist needs $4-6$ sessions CBT. And in accord with our previous study [24], we excluded trials if they met the following criteria: the duration of treatment was less than 6 weeks, or the number of sessions was less than 6 . Finally, studies were excluded if we detected repeated publication.

The inclusion process was conducted by two independent reviewers (BY and TT). First, the reviewers scanned the abstract and title of potential papers, and identified studies to be read in full text. The final selection of studies was conducted by both reviewers. If there was any disagreement between the two reviewers, another reviewer was consulted to resolve the discrepancy (XZ).

\section{Outcome measures}

The primary outcome of efficacy was the mean change scores of the anxiety rating scale from baseline to posttreatment. When there was more than one available anxiety rating scale in one study, we used the scores from the anxiety rating scale according to a predefined hierarchy [24], based on psychometric properties and frequency for use with children and adolescents. We also established a hierarchy of informants of anxiety rating scales, with the child or adolescent self-report first in the hierarchy, then the parent/teacher report and then the clinician report. Finally the hierarchy of anxiety symptom severity measurement scales are as below: (1) Revised Children's Manifest Anxiety Scale (RCMAS), (2) Spence Child Anxiety Scale, Child and Parent Versions (SCAS), (3) Screen for Child Anxiety-Related Emotional Disorders (SCARED), (4), Clinician severity ratings (CSR) (5) Fear Survey for Children Revised (FSSC-R), (6) Child Behavior Checklist (CBCL), (7) other scale.

The second efficacy outcome was remission rate measured by the proportion of participants who did not meet the standardized diagnostic criteria for anxiety disorder after treatment.

The outcome of acceptability was all-cause discontinuation, defined as the proportion of patients in the treatment group who withdrew from treatment early for any reason.

\section{Data extraction and methodological quality}

Two independent reviewers (BY and TT) used standardized data extraction forms to extract data of the main characteristics of all trials, and the methodological qualities of trials were also assessed. Standardized data extraction forms included data on study characteristics (e.g., publication year, first listed author, country, journal, sponsor, institution), intervention details (e.g., duration of treatment, session of treatment, treatment pattern), patients' characteristics (the number of patients, diagnostic criteria for anxiety disorders), and outcome measures (e.g., remission rate, pre- and posttreatment outcomes). The risk of bias among all studies was assessed according to the Cochrane Collaboration Risk of bias tool described in the Cochrane Handbook for Systematic Reviews of Intervention [25]. If there was any disagreement between the two reviewers, $\mathrm{XZ}$ was consulted to resolve the discrepancy. We did not determine the risk of bias across studies, because the number of included studies was too small.

\section{Statistical analysis}

We compared the relative efficacy and acceptability by performing a meta-analysis using Review Manager 5.3 (The Cochrane Collaboration of The Nordic Cochrane Center in Copenhagen). Because of various anxiety rating scales among included studies, there maybe exist underlying heterogeneity and the difference in the true treatment effect among studies. So the DerSimonian and Laird random-effects models were adopted for all metaanalyses [26].

Pooled estimates of standardized mean difference (SMD) were calculated with 95\% confidence intervals (CIs) for continuous outcomes. An SMD of more than 0 indicates that the comparison conditions (WL or CBT with parents) were more effective. Conversely, an SMD value of less than 0 indicates that the parent-only CBT condition was more effective. Regarding the risk ratios (RRs), we calculated 95\% confidence intervals for discontinuous outcomes. Values of more or less than 1 indicate that the comparison groups or parent-only CBT group more frequently involved events of concern, respectively. Additionally, heterogeneity of both effects among studies was assessed using the $p$-values of the $\mathrm{Q}$ statistic and $\mathrm{I}^{2}$ statistic. For the primary outcome of efficacy(parent-only CBT compared with WL), we realized significant heterogeneity $\left(\mathrm{I}^{2}=81 \%, P=0.001\right)$. Then we performed sensitivity analysis by excluding the outlier, the study of Cartwright-Hatton (2011) in forest plot Fig. 2a.

To test significant differences in primary efficacy between the different categories of studies, we also performed subgroup analyses. The studies were divided into different categories based on the following factors: male to female ratio (more than 1 or not more than 1), the 
risk of bias according to the Cochrane Collaboration Risk of bias tool (high bias risk or unclear risk) and the anxiety rating scale (other-rated scale or self-rated scale), respectively.

As for publication bias, we performed Egger's teston STATA.

\section{Results}

\section{Study selection and characteristics}

After searching six electronic databases, we identified 6878 potentially relevant studies. Then, 118 full-text articles were identified for review. Finally, six RCTs with 407 participants were included in our meta-analyses. A flow diagram showing the details of the inclusion and exclusion of studies is presented in Fig. 1. And more details of exclusion can be found in Additional file 2.

Detailed information about the included studies is presented in Table 1 and Additional file 3. There were four studies of parent-only CBT compared with WL, and three studies of parent-only CBT vs. CBT with parents. In total, we included 199 patients in the parent-only CBT group, 114 patients in the WL group and 94 patients in the CBT with parents group. The mean age of all patients recruited was 8.0 years old.

\section{Efficacy outcome}

For the primary outcome of efficacy, we first compared the parent-only CBT with the WL control group. The overall pooled SMD indicated a significant advantage when parent-only CBT was compared with $\mathrm{WL}$, with SMD of $-0.72(95 \%$ CI -1.41 to $-0.03, p=0.04,4$ studies including 206 patients, Egger's test $P=0.787$ ) and high heterogeneity $\left(\mathrm{I}^{2}=81 \%, p=0.001\right.$ Fig. 2a). We then compared parent-only with CBT with parents, with SMD of 0.21 (95\% CI -0.09 to $0.50, p=0.17,3$ studies including 178 patients, Egger's test $P=0.339$ ) and low heterogeneity $\left(\mathrm{I}^{2}=0 \%, p=0.89\right.$ Fig. $\left.2 \mathrm{~b}\right)$.

For the secondary outcome of efficacy, only 3 studies including 181 patients reported information about remission rate. All three of these studies belonged to the WL control group. Patients receiving parent-only CBT were more likely to report remission than those receiving WL, with RR of 4.33 (37.96\% vs. 6.85, 95\% CI 1.82 to 10.27, $p=0.0009$, Egger's test $P=0.295$ ) and low heterogeneity $\left(\mathrm{I}^{2}=0 \%, p=0.44\right.$ Fig. 3$)$.

\section{Acceptability outcomes}

In terms of the acceptability outcome, we found no significant difference between the parent-only intervention group and the WL group. The all-cause discontinuation rates were $30 / 146(20.55 \%)$ for the parent-only intervention group (4 studies), and 20/114 (17.54\%) for the WL group (4 studies). There was no significant difference between the two groups. The RR value was 0.92 (95\% CI

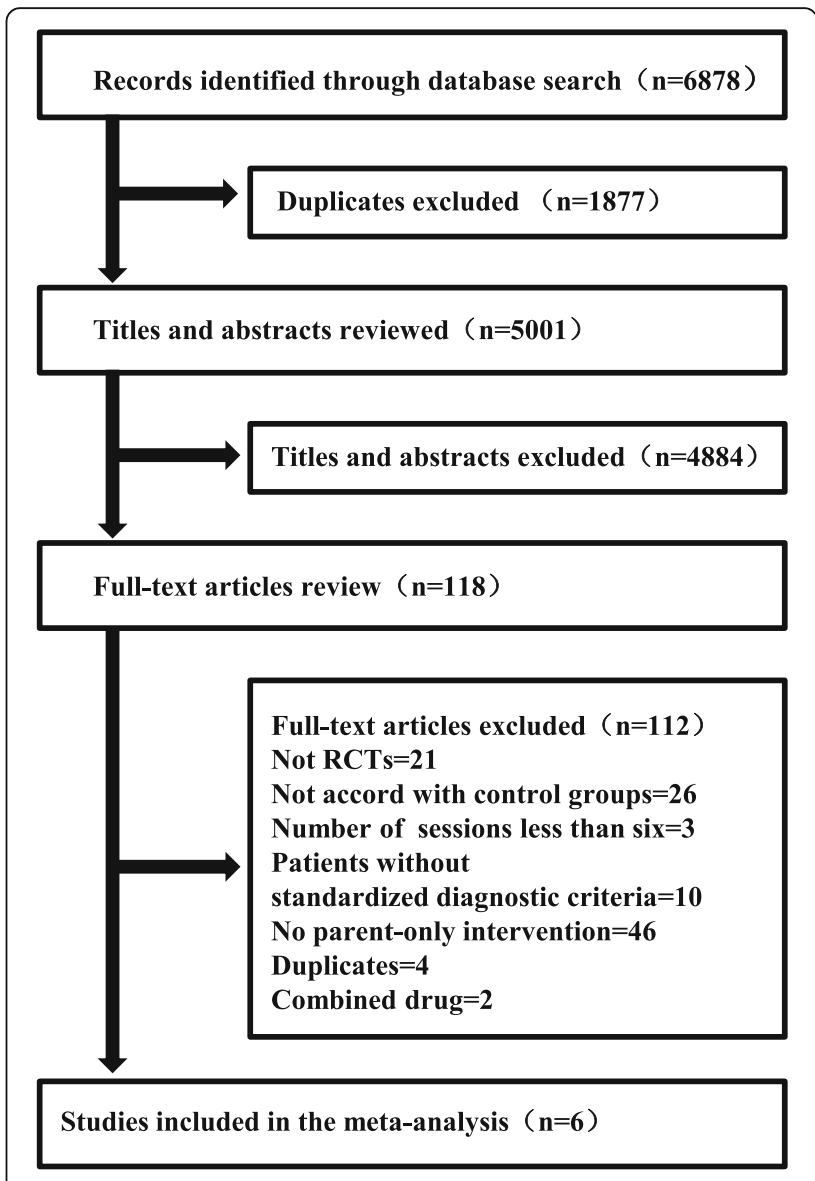

Fig. 1 Flowchart of study selection

0.52 to $1.62, p=0.77$, Egger's test $P=0.342$ ) with low heterogeneity $\left(\mathrm{I}^{2}=3 \%, p=0.38\right.$ Fig. $\left.4 \mathrm{a}\right)$. Regarding acceptability between parent-only and CBT with parents, the all-cause discontinuation rates were 23/91 (25.27\%) for the parent-only group(3 studies), and 12/94 (12.77\%) for the CBT with parents group (3 studies). The RR value was 1.93 ( $95 \%$ CI 1.05 to $3.57, p=0.03$ ) with low heterogeneity $\left(\mathrm{I}^{2}=0 \%, p=0.33\right.$ Fig. $\left.4 \mathrm{~b}\right)$.

\section{Sensitivity analysis}

We performed sensitivity analysis by excluding the study of Cartwright-Hatton (2011), where the effect size greatly changed $(\mathrm{SMD}=-1.05 ; 95 \% \mathrm{CI}-1.40$ to -0.71 , ; $P<0.00001)$, with low heterogeneity $\left(\mathrm{I}^{2}=0 \%, p=0.93\right.$ Fig. 5).

\section{Subgroup analysis}

We conducted subgroup analysis according to the proportion of male/female patients included in the studies. The results revealed no significant differences between two outcomes of this subgroup analysis $(p=0.43)$. Studies with more girls (male/female $\leq 1)$ did not show a greater effect size than WL $(\mathrm{SMD}=-0.41,95 \% \mathrm{CI}-$ 


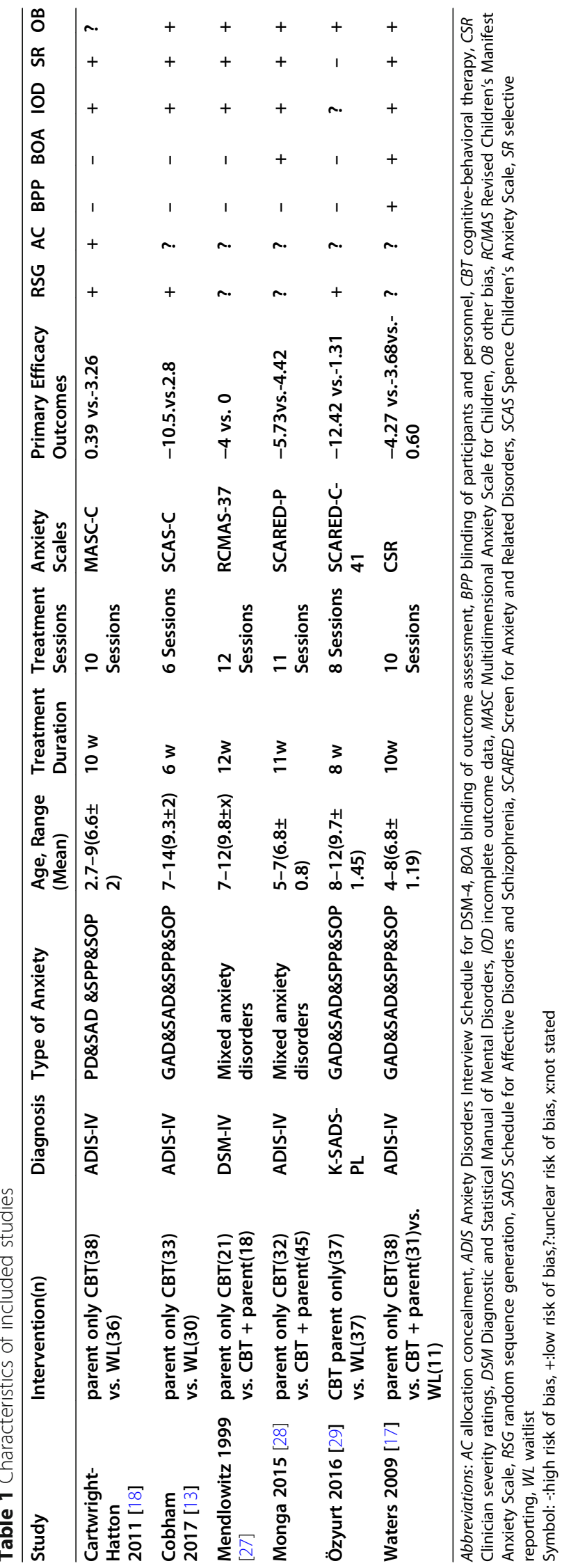




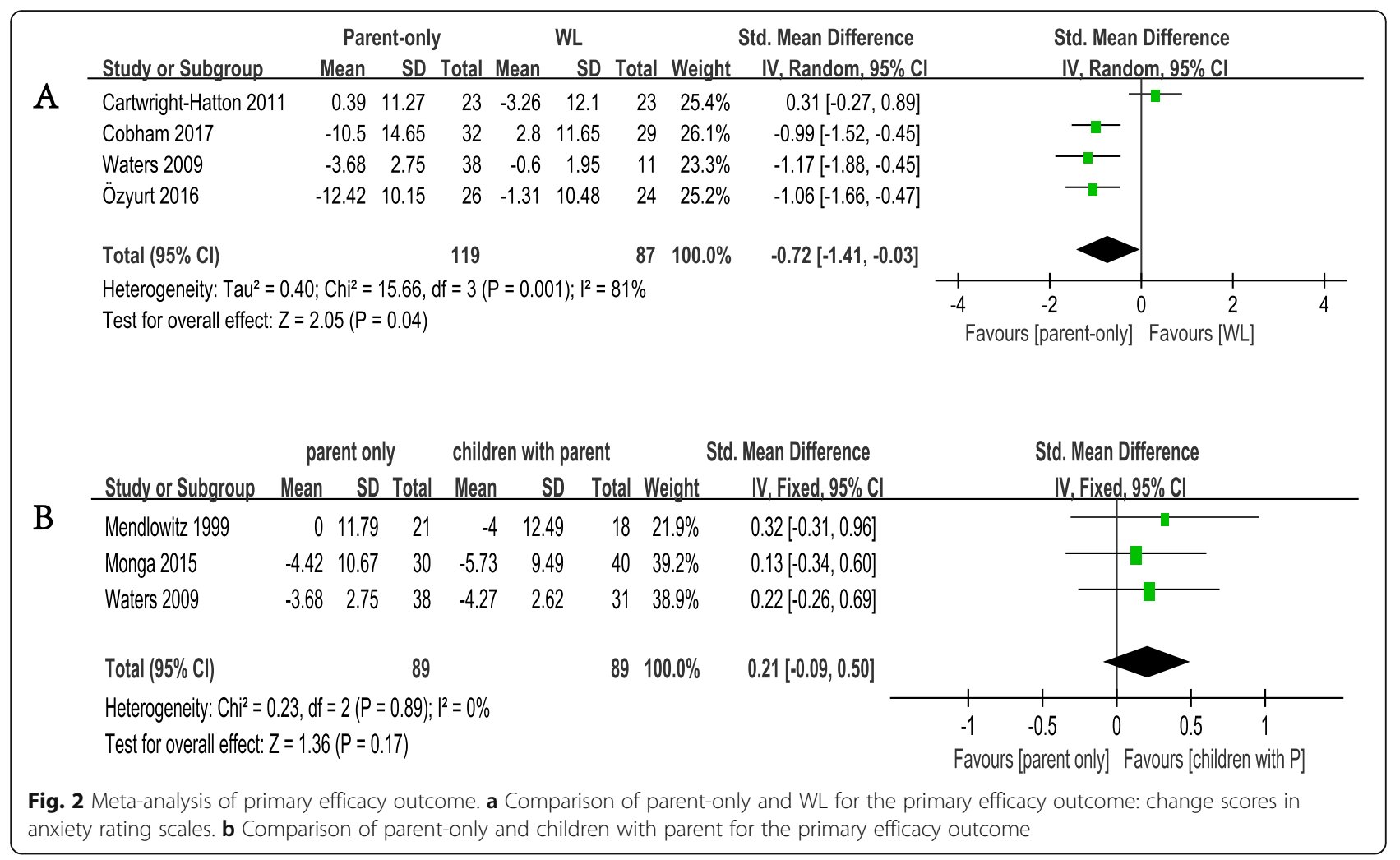

1.86 to $1.03 ; p=0.57$ ), but studies with more boys (male/female $>1$ ) showed a significant difference between two groups (SMD $=-1.02,95 \% \mathrm{CI}-1.42$ to 0.62; $p<0.00001$ Fig. 6a).

We found no significant differences between studies in which anxiety was measured using different rating scales $(p=0.31)$. When we compared parent-only CBT with WL. Studies that included patients measured with otherrated scales $(\mathrm{SMD}=-1.17,95 \% \mathrm{CI}-1.88$ to $-0.45 ; p=$ $0.001)$ still appeared to be more effective. Studies that recruited patients measured with self-rated measures exhibited no significant differences (SMD $=-0.58,95 \% \mathrm{CI}$ - 1.44 to $0.28 ; p=0.18$ Fig. $6 b)$.

Furthermore, for comparing parent-only CBT with WL, we divided previous studies into two subgroups by the risk of bias (high bias risk or unclear risk). We found no significant differences $(p=0.41)$ between studies with high risk $(\mathrm{SMD}=-1.06,95 \% \mathrm{CI}-1.66$ to $-0.47 ; p=$ $0.0005)$ and unclear risk $(\mathrm{SMD}=-0.61,95 \% \mathrm{CI}-1.52$ to $0.31 ; p=0.19$ Fig. $6 \mathrm{c}$ ).

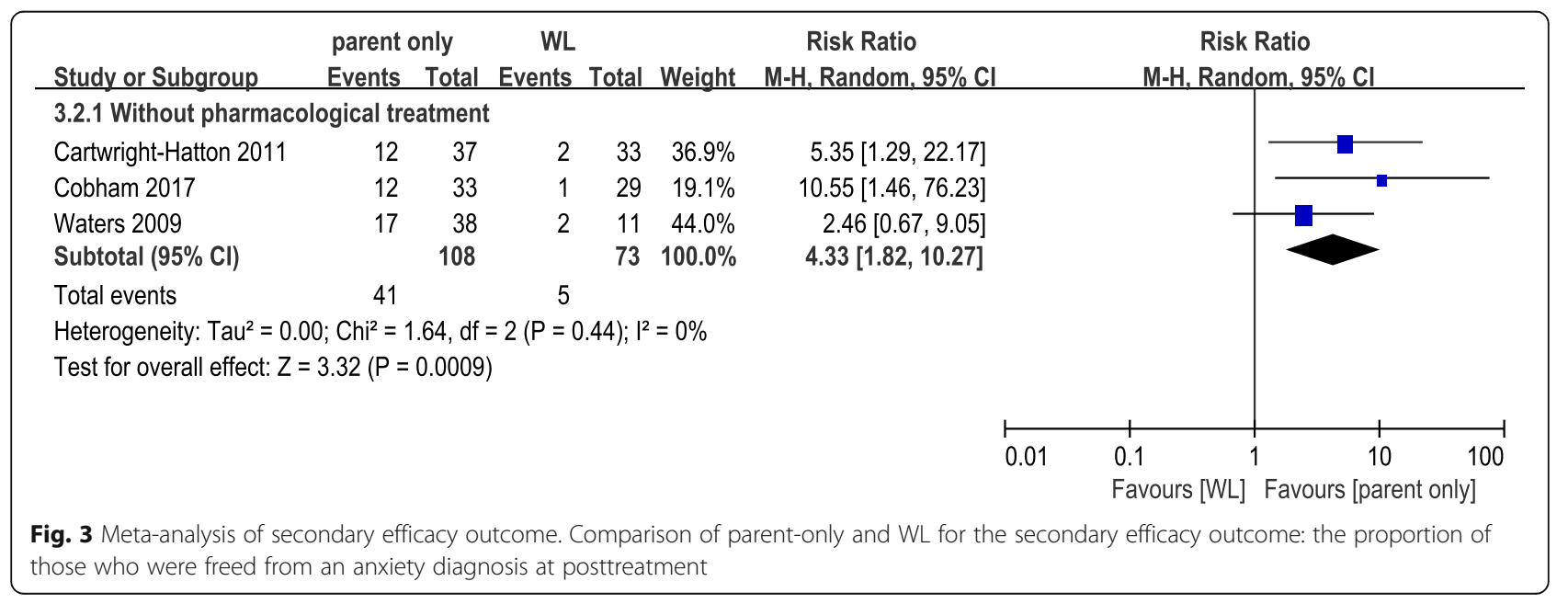




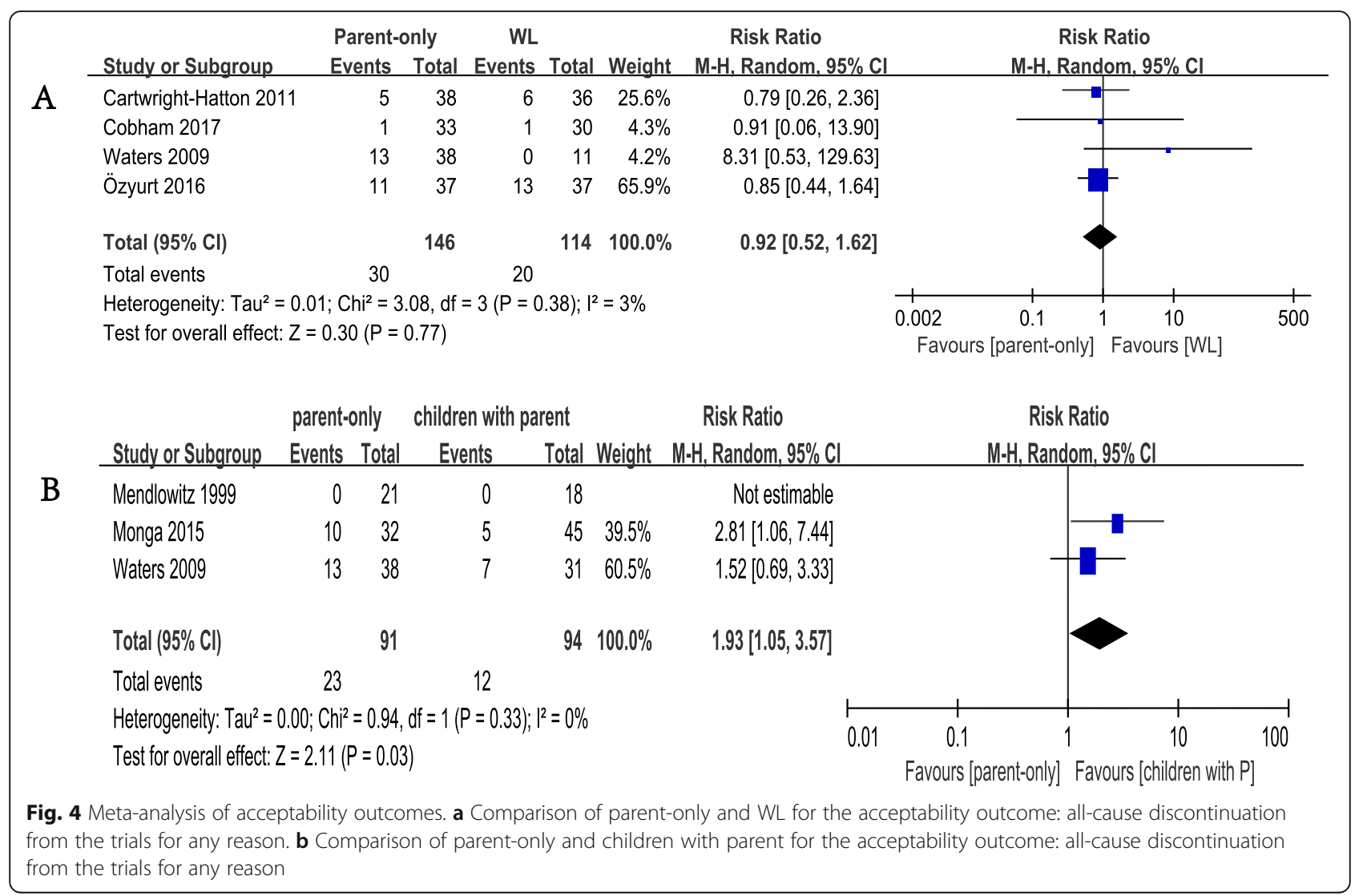

\section{Quality assessment}

In total, the quality of included studies was relatively moderate, and the quality of studies met the standard for high risk of bias in several studies. There were three studies with a low risk of bias owing to random sequence generation. Only one study reported a low risk of bias owing to allocation concealment and performance bias. Two studies had a low risk of bias owing to detection bias. Five studies had a low risk of bias owing to reporting bias, attrition bias and other bias. The details of risk of bias are presented in Table 1 and Additional file 4 .

\section{Discussion}

Anxiety disorder is a severe disease among children and adolescents, which can threaten academic and social competence $[2,3]$. Parent-only CBT is an important and novel treatment for anxiety disorder in children and adolescents. In the current study, we identified RCTs of parent-only CBT for analysis. Given the limited number of included studies, we sought to enhance the validity of our conclusions by conducting two associative pair-wise meta-analyses, including a WL control group, and comparing groups undergoing CBT with parents, and parent-only CBT. In both of the comparison groups, we assessed efficacy using mean change scores on the anxiety rating scale from baseline to post-treatment. Acceptability was represented by the proportion of participants who did not meet the standardized diagnostic criteria of anxiety disorders when treatment was finished. We then assessed remission rate and conducted subgroup analysis in the control group only.

Regarding efficacy, the results suggested that, compared with the WL control condition, parent-only CBT is an effective treatment for reducing anxiety symptoms and relieving anxiety in children, leading to remission at the end of treatment. The findings in this pair-wise meta-analysis were consistent with a previously reported network meta-analysis [30]. The network meta-analysis included studies in which more than $20 \%$ of children took psychotropic drugs, and revealed that parent-only CBT led to better outcomes than WL control condition in children with various types of anxiety disorders. Comparison revealed parent-only CBT has mild weakly (without significant difference) efficacy than CBT involving parents. According to previous studies [30, 31], CBT involving parents was effective for treating child anxiety disorders. Particularly for early childhood anxiety, CBT involving parents is reported to be more beneficial for young children than for older children because of their limited language and cognitive competence [32]. In the current study, parent-only CBT had the mild weakly 


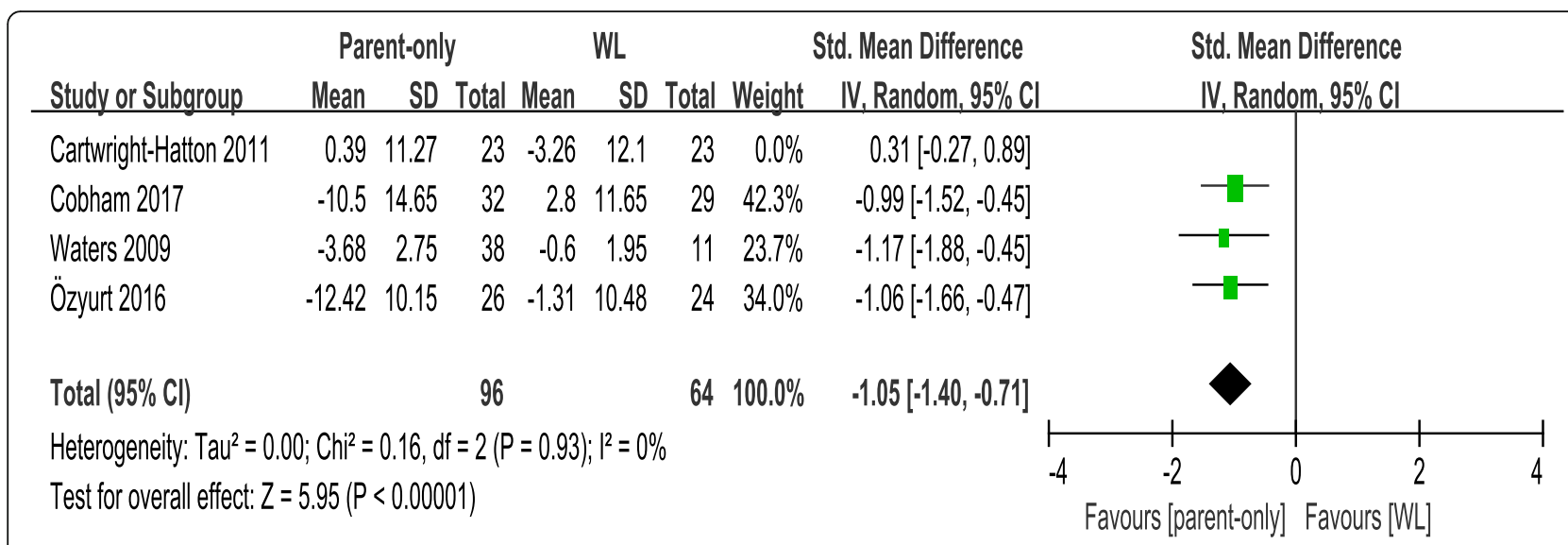

Fig. 5 sensitivity analysis which excluded the study of Cartwright-Hatton (2011) [18]

(without significant difference) efficacy than CBT with parents, and was efficacious compared with the WL condition. Previous study [10] which reported child-CBT remission rate $(48.47 \%)$ supported the remission of parent-only CBT (37.96\%)was mild weak than childCBT. And our results regarding the two comparison groups were consistent and in accord other child-CBT (without significant difference), suggesting that parentonly CBT is one of effective treatments for anxiety disorder in children and adolescents.

To determine the influence of various conditions on the primary efficacy outcome, we conducted subgroup analysis of male/female, high/unclear risk and self/otherrated patients, between the parent-only and WL groups. Regarding male/female differences, the results revealed more significant improvements in boys than girls. An early study of 79 children with anxiety in 1996 reported gender differences in the way parents interacted with anxious children, indicating that younger, female children benefitted more from parental involvement [33]. It is inconsistent with our finding. Therefore confirming this finding will require further research in future. Regarding high/unclear risk, excluding one high-risk study [29] changed the efficacy of the outcome. This finding highlighted the need for caution when interpreting the current results, and the importance of further studies to confirm our conclusions. When subgroups depended on self/other-rating scales, excluding one study [17] that measured anxiety in children using an other-rated scale (clinical severity rating, CSR, doctor-rated) resulted in different effects. This result may related to overstatement of improvement of anxiety symptoms by doctors. According to a previous study using three kinds of rating scales (doctor-rated, parent-rated and child-rated) to measure anxiety in the same children, the doctor-rated scale produced the least similar results among the three kinds of rating scales, while the parent-rated scale showed smaller differences than the doctor-rated scale, and the self-rated scale showed no differences [34]. This phenomenon is consistent with our speculation that findings may be overstated when doctorrated scales are used to examine children's anxiety.

Interestingly, for the primary outcome of efficacy(parent-only CBT compared with WL), we realized significant heterogeneity. Then we performed sensitivity analysis. We excluded the study of Cartwright-Hatton (2011), which made heterogeneity fromdwon to and $p$ value from to. We investigated deeply into the study of Cartwright-Hatton (2011). we found that the children relatively young and MASC was adopted. But MASC was developed for children 8 years and older. The selfrated scale MASC may do not inappropriate to anxiety in young children. For example, the studies (Waters 2009 and Monga 2015) included young children adopted other-rated scale.

Regarding acceptability, we found no significant differences between the parent-only CBT and WL conditions. However, more families tended to drop out of treatment in the parent-only group compared with the CBT with parents group. This result is similar to those of other studies investigating internet-based delivery and bibliotherapy as alternative modes of CBT treatment [35-37]. It is possible that parents did not have sufficient trust in the efficacy of parent-only CBT because their children were not directly involved in the treatment, resulting in a tendency to drop out early.. And the results of a previous study suggest that the additional responsibility in the parent-only condition may explain the tendency to drop out in the parent-only condition. This explanation supports the importance of assessing the level of sense of responsibility of parents and enhancing it prior to commencing treatment [17]. However, the conclusions that can be drawn from the current findings regarding acceptability are limited due to the small sample size.

In the current study, we did not investigate the effects of parent-only CBT in young children and older 


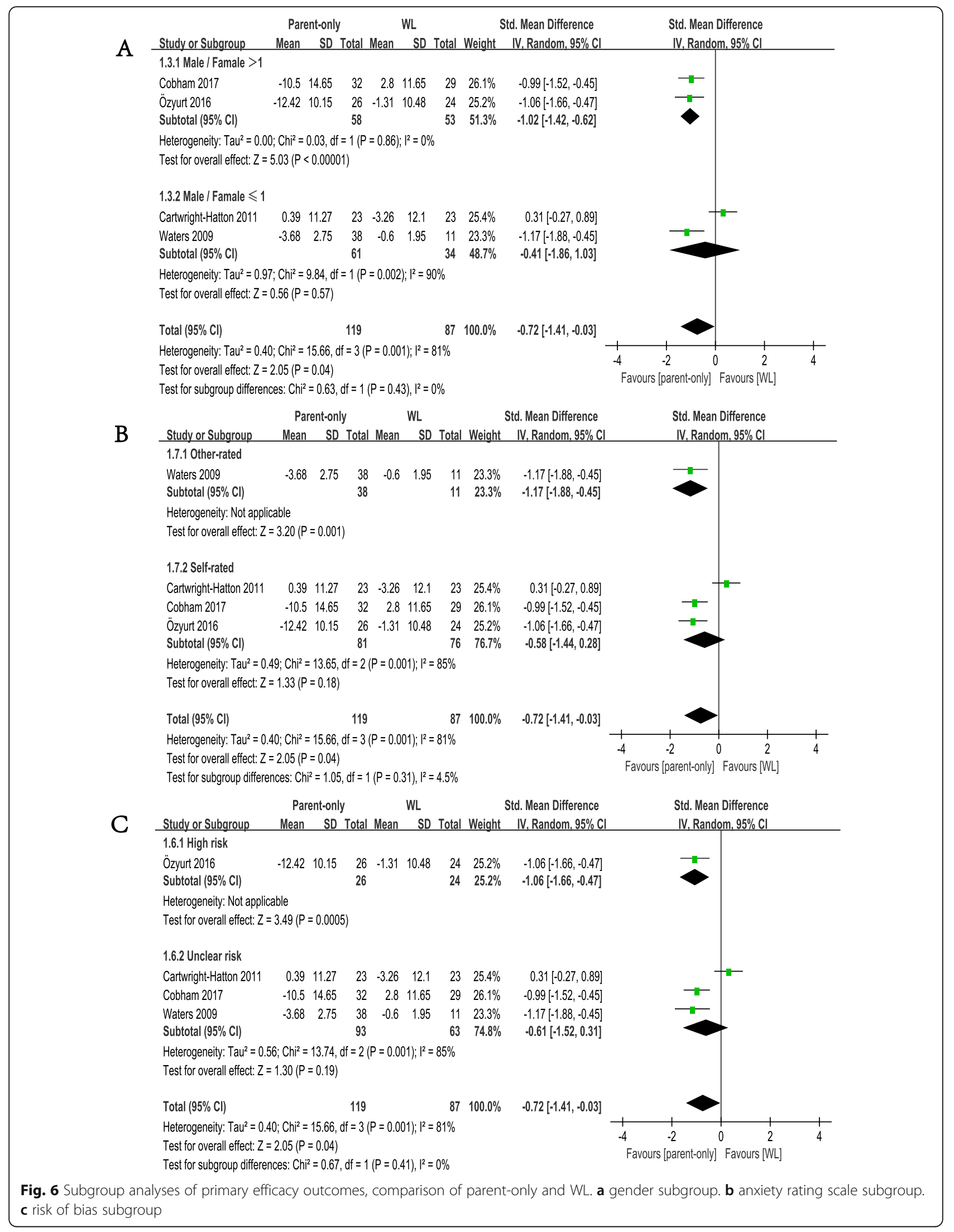


children, respectively, due to a lack of studies. According to previous studies of CBT with parents in young children [32], family-based CBT in which parents are highly involved is a well-established effective intervention for early childhood anxiety. Thus, future studies should investigate whether parent-only CBT is a similarly effective treatment, which could be more beneficial for young children than the older. Moreover, in the etiology of childhood anxiety, the influence of parents' own anxiety levels is important and well recognized [38]. In the current meta-analysis, only two studies reported scale score change of parents' own anxiety levels from pretreatment to post-treatment. Waters reported that parents with high anxiety scores were more likely to drop out, both in the intervention group and the control group, but only a non-significant trend was observed on the DASS-42 Anxiety subscales from pre-treatment to post-treatment [17]. And Ozyurt reported a significant improvement in parental anxiety in the intervention group from pre-treatment to post-treatment [29]. No previous studies have explored the differences between implementing parent-only CBT for children with anxiety when parents met the diagnostic criteria for anxiety or depression. Elucidating these issues will require more RCTs in future.

The current study involved several limitations that should be considered. First, the sample size was not sufficient to ensure reliable statistical power, particularly in subgroup analyses. In addition, the unsatisfactory quality of some of the included studies limited the reliability of the conclusions, and the studies included in the review exhibited substantial heterogeneity. Therefore, caution is required when interpreting the current findings. Moreover, other types of CBT involving parental participation were not taken into consideration, such as parentdelivered CBT, internet-delivered CBT with parents, and telephone-delivered CBT with parents. We did not investigate the efficacy of parent-only CBT for specific types of anxiety in children. Elucidating these issues will require further investigation. Due to a lack of data in the included studies, we did not examine follow-up assessment results. Resolving this shortcoming will require future studies.

\section{Conclusions}

Our meta-analysis suggests that parent-only CBT is one of effective interventions for children with anxiety disorder. Although parent-only CBT increased the rate of all-cause discontinuation compared with CBT with parents, it did not lead to more patients dropping out compared with the WL control group. Current evidence indicates that parent-only CBT is both an acceptable and alternative treatment compared with a control condition for children and adolescents with anxiety disorder.

\section{Supplementary Information}

The online version contains supplementary material available at https://doi. org/10.1186/s12888-020-03021-0.

Additional file 1: Table S1-S6. Literature search report: Cochrane (613), Embase (386), ProQuest (810), PsycINFO (559), PubMed (1393), Web of

Science (3118) and Additional file 2

Additional file 2. 112 excluded studies, and the reasons they were excluded.

Additional file 3. Data extraction forms.

Additional file 4. Graphical overview of the methodological quality of included studies.

\section{Abbreviations}

AC: Allocation concealment; ADIS: Anxiety Disorders Interview Schedule for DSM-4; BOA: Blinding of outcome assessment; BPP: Blinding of participants and personnel; $\mathrm{CBCL}$ : Child Behavior Checklist; CBT: Cognitive behavioral therapy; Cls: Confidence intervals; CSR: Clinician severity ratings;

DASS: Depression Anxiety Stress Scale; DSM: Diagnostic and Statistical Manual of Mental Disorders; FSSC-R: Fear Survey for Children Revised; IOD: Incomplete outcome data; MASC: Multidimensional Anxiety Scale for Children; OB: Other bias; RCMAS: Revised Children's Manifest Anxiety Scale; RCT: Randomized controlled trial; RRs: Risk ratios; RSG: Random sequence generation; SADS: Schedule for Affective Disorders and Schizophrenia; SCAR ED: Screen for Anxiety and Related Disorders; SCAS: Spence Children's Anxiety Scale; SMD: Standardized mean difference; SPAI-C: Social Phobia and Anxiety Inventory for Children; SR: Selective reporting; WL: Waitlist

\section{Acknowledgments}

N/A

\section{Authors' contributions}

The author (PX \& XZ) conceptualized the study and approved the final manuscript. BY conducted analyses, write-up, quality assessment, write the discussion and draft the final manuscript. $\Pi$ and LT participated in analyses, data extraction and quality assessment. $\mathrm{XL}$ and LF participated in search, data extraction, analyses and interpretation of data preliminarily. All authors read and approved the final manuscript.

\section{Funding}

Science and Technology Research Project of Chongqing Education Commission, KJQN201800415.

High-end R\&D Talent Support Project of Chongqing Science and Technology Bureau, cstc2018kjcxljrc0038

The funding body subsidizes the cost of office space and necessary facilities.

Availability of data and materials

All data generated or analyzed during this study are included in this article.

Ethics approval and consent to participate

N/A

Consent for publication

N/A

Competing interests

The authors declare that they have no competing interests.

\section{Author details}

${ }^{1}$ Department of Neurology, People's Hospital of Deyang City, Deyang, China. 2Department of Neurology, The First Affiliated Hospital of Chongqing Medical University, Chongqing, China. ${ }^{3} \mathrm{NHC}$ Key Laboratory of Diagnosis and Treatment on Brain Functional Diseases, The First Affiliated Hospital, Chongqing Medical University, No. 1 Youyi Road, Chongqing 400016, China. ${ }^{4}$ Department of Immunology, College of Basic Medical Sciences, China Medical University, Shenyang, China. ${ }^{5}$ Department of Psychiatry, The First Affiliated Hospital of Chongqing Medical University, No. 1 Youyi Road, Chongqing 400016, China. 
Received: 12 January 2020 Accepted: 22 December 2020

Published online: 11 January 2021

\section{References}

1. Polanczyk GV, Salum GA, Sugaya LS, Caye A, Rohde LA. Annual research review: a meta-analysis of the worldwide prevalence of mental disorders in children and adolescents. J Child Psychol Psychiatry. 2015;56(3):345-65.

2. Mychailyszyn M, Mendez JC, Kendall P. School functioning in youth with and without anxiety disorders: comparisons by diagnosis and comorbidity. Sch Psychol Rev. 2010;39:106-21.

3. Wood JJ, Piacentini JC, Southam-Gerow M, Chu BC, Sigman M. Family cognitive behavioral therapy for child anxiety disorders. J Am Acad Child Adolesc Psychiatry. 2006:45(3):314-21.

4. Cartwright-Hatton S, Roberts C, Chitsabesan P, Fothergill C, Harrington R. Systematic review of the efficacy of cognitive behaviour therapies for childhood and adolescent anxiety disorders. Br J Clin Psychol. 2004;43(Pt 4): 421-36.

5. In-Albon T, Schneider S. Psychotherapy of childhood anxiety disorders: a meta-analysis. Psychother Psychosom. 2007;76(1):15-24.

6. James AC, James G, Cowdrey FA, Soler A, Choke A. Cognitive behavioural therapy for anxiety disorders in children and adolescents. Cochrane Database Syst Rev. 2015;2:CD004690.

7. James AC, James G, Cowdrey FA, Soler A, Choke A. Cognitive behavioural therapy for anxiety disorders in children and adolescents. Cochrane Database Syst Rev. 2013;6:CD004690.

8. Seligman LD, Ollendick TH. Cognitive-behavioral therapy for anxiety disorders in youth. Child Adolesc Psychiatr Clin N Am. 2011;20(2):217-38.

9. Walkup JT, Albano AM, Piacentini J, Birmaher B, Compton SN, Sherrill JT, Ginsburg GS, Rynn MA, McCracken J, Waslick B, et al. Cognitive behavioral therapy, sertraline, or a combination in childhood anxiety. N Engl J Med. 2008;359(26):2753-66.

10. Zhang $H$, Zhang $Y$, Yang L, Yuan S, Zhou X, Pu J, Liu L, Jiang X, Xie PJTJ, disease m. Efficacy and Acceptability of Psychotherapy for Anxious Young Children: A Meta-analysis of Randomized Controlled Trials. 2017; 205(12):931-41.

11. Reynolds S, Wilson C, Austin J, Hooper L. Effects of psychotherapy for anxiety in children and adolescents: a meta-analytic review. Clin Psychol Rev. 2012;32(4):251-62.

12. Thulin U, Svirsky L, Serlachius E, Andersson G, Ost LG. The effect of parent involvement in the treatment of anxiety disorders in children: a metaanalysis. Cogn Behav Ther. 2014;43(3):185-200.

13. Cobham VE, Filus A, Sanders MR. Working with parents to treat anxietydisordered children: a proof of concept RCT evaluating fear-less triple P. Behav Res Ther. 2017:95:128-38.

14. Dempster $\mathrm{R}$, Wildman $\mathrm{B}$, Keating $\mathrm{A}$. The role of stigma in parental helpseeking for child behavior problems. J Clin Child Adolescent Psychology. 2013;42(1):56-67.

15. Rapee RM, Bogels SM, van der Sluis CM, Craske MG, Ollendick T. Annual research review: conceptualising functional impairment in children and adolescents. J Child Psychol Psychiatry. 2012;53(5):454-68.

16. Salari E, Shahrivar Z, Mahmoudi-Gharaei J, Shirazi E, Sepasi M. Parent-only Group Cognitive Behavioral Intervention for Children with Anxiety Disorders: A Control Group Study. J Can Acad Child Adolescent Psychiatry. 2018;27(2):130-6.

17. Waters AM, Ford LA, Wharton TA, Cobham VE. Cognitive-behavioural therapy for young children with anxiety disorders: comparison of a child + parent condition versus a parent only condition. Behav Res Ther. 2009;47(8):654-62.

18. Cartwright-Hatton S, McNally D, Field AP, Rust S, Laskey B, Dixon C, Gallagher B, Harrington R, Miller C, Pemberton K, et al. A new parentingbased group intervention for young anxious children: results of a randomized controlled trial. J Am Acad Child Adolesc Psychiatry. 2011;50(3): 242-51 e246.

19. Moher D, Liberati A, Tetzlaff J, Altman DG. Preferred reporting items for systematic reviews and meta-analyses: the PRISMA statement. PLoS Med 2009;6(7):e1000097.

20. Rabe-Jablonska J. A new draft of the mental disorders classification prepared by the American Psychiatric Association: diagnostic and statistical manual of mental disorders-IV, options book. Psychiatr Pol. 1993;27(2):109-19.

21. Silverman WK, Nelles WB. The anxiety disorders interview schedule for children. J Am Acad Child Adolesc Psychiatry. 1988;27(6):772-8.
22. Kaufman J, Birmaher B, Brent D, Rao U, Flynn C, Moreci P, Williamson D, Ryan N. Schedule for affective disorders and schizophrenia for school-age children-present and lifetime version (K-SADS-PL): initial reliability and validity data. J Am Acad Child Adolesc Psychiatry. 1997; 36(7):980-8.

23. Robinson PJ, Strosahl KD. Behavioral health consultation and primary care: lessons learned. J Clin Psychol Med Settings. 2009;16(1):58-71.

24. Zhang $Y$, Zhou $X$, James AC, Qin B, Whittington CJ, Cuijpers P, Del Giovane C, Liu Y, Cohen D, Weisz JR, et al. Comparative efficacy and acceptability of psychotherapies for acute anxiety disorders in children and adolescents: study protocol for a network meta-analysis. BMJ Open. 2015;5(10):e008572

25. Cumpston M, Li T, Page MJ, Chandler J, Welch VA, Higgins JP, Thomas J. Updated guidance for trusted systematic reviews: a new edition of the Cochrane handbook for systematic reviews of interventions. Cochrane Database Syst Rev. 2019;10:ED000142.

26. Borenstein M, Hedges LV, Higgins JP, Rothstein HR. A basic introduction to fixed-effect and random-effects models for meta-analysis. Res Synth Methods. 2010;1(2):97-111.

27. Mendlowitz SL, Manassis K, Bradley S, Scapillato D, Miezitis S, Shaw BF. Cognitive-behavioral 4 group treatments in childhood anxiety disorders: the role of parental involvement. J Am Acad Child Adolesc Psychiatry. 1999; 38(10):1223-9.

28. Monga S, Rosenbloom BN, Tanha A, Owens M, Young A. Comparison of child-parent and7 parent-only cognitive-behavioral therapy programs for anxious children aged 5 to 7 years: 8 short- and long-term outcomes. J Am Acad Child Adolesc Psychiatry. 2015;54(2):138-46.

29. Ozyurt. Is triple P positive parenting program effective on anxious children and their parents? 4th month follow up results. J Child Fam Stud. 2016; 25(5):1646.

30. Zhou X, Zhang Y, Furukawa TA, Cuijpers P, Pu J, Weisz JR, Yang L, Hetrick SE, Del Giovane C, Cohen D, et al. Different types and acceptability of psychotherapies for acute anxiety disorders in children and adolescents: a network meta-analysis. JAMA Psychiatry. 2019;76(1):41-50.

31. Kreuze LJ, Pijnenborg GHM, de Jonge YB, Nauta MH. Cognitive-behavior therapy for children and adolescents with anxiety disorders: a meta-analysis of secondary outcomes. J Anxiety Disord. 2018;60:43-57.

32. Comer JS, Hong N, Poznanski B, Silva K, Wilson M. Evidence Base Update on the Treatment of Early Childhood Anxiety and Related Problems. J Clin Child Adolescent Psychology. 2019;48(1):1-15.

33. Barrett PM, Dadds MR, Rapee RM. Family treatment of childhood anxiety: a controlled trial. J Consult Clin Psychol. 1996;64(2):333-42.

34. Vigerland S, Ljotsson B, Thulin U, Ost LG, Andersson G, Serlachius E. Internetdelivered cognitive behavioural therapy for children with anxiety disorders: a randomised controlled trial. Behav Res Ther. 2016;76:47-56.

35. Lyneham HJ, Rapee RM. Evaluation of therapist-supported parentimplemented CBT for anxiety disorders in rural children. Behav Res Ther 2006;44(9):1287-300

36. Rapee RM, Abbott MJ, Lyneham HJ. Bibliotherapy for children with anxiety disorders using written materials for parents: a randomized controlled trial. J Consult Clin Psychol. 2006;74(3):436-44.

37. Spence SH, Holmes JM, March S, Lipp OV. The feasibility and outcome of clinic plus internet delivery of cognitive-behavior therapy for childhood anxiety. J Consult Clin Psychol. 2006;74(3):614-21.

38. Craske MG, Waters AM. Panic disorder, phobias, and generalized anxiety disorder. Annu Rev Clin Psychol. 2005;1:197-225.

\section{Publisher's Note}

Springer Nature remains neutral with regard to jurisdictional claims in published maps and institutional affiliations. 